\title{
Microstructure of Fish Meat Emulsion with Addition of Egg-Yolk
}

\author{
Teruo NAKAYAMA and Hiroko TOMITA \\ Faculty of Bioresources, Mie University, 1515, Kamihama-cho, Tsu, Mie 514, Japan
}

Received September 3, 1996

\begin{abstract}
Fish meat emulsion was prepared from egg-yolk, very low-lipid sardine meat (which was prepared through grinding or suspending in weak alkaline solution), salad oil, and vinegar. The microstructures of the oil droplet surface and myofibrillar matrix were investigated. By Polytron homogenization, the myofibrils were shattered to a much smaller size in ground-meat emulsion, according to the original smaller fragment size. The network structure was formed by the myofilaments spread apart from the shattered myofibrils in the fish meat emulsion. The mesh size of this structure was smaller in the ground-meat emulsion than in the suspended-meat one and corresponded to the oil droplet size. In the suspended-meat emulsion, the oil droplet size was smaller at oil ratio 1.1 to sardine meat than at the ratio 1.6. In the non-fish conventional emulsion, the viscosity increase due to the dispersion of oil droplets to the egg-yolk matrix was not sufficient to prevent irreversible shear breakdown induced by coalescence. In fish meat emulsion, the network structure made of myofilaments showed a remarkable increase in viscosity and held the oil droplets tightly during the application of high shear rates to prevent the coalescence and exhibit a weak trend of thixotropy.
\end{abstract}

Keywords: fish meat emulsion, microstructure, egg-yolk, oil droplet, myofibrillar matrix

Nutritional substances are richly contained in sardine meat. In our previous study (Nakayama et al, 1996), a fish meat emulsion was prepared from egg-yolk, very low-lipid sardine minced meat (which was prepared through grinding or suspending in weak alkaline solution), salad oil, and vinegar. This emulsion was evaluated to be fishy-odorless, cream-colored, and excellent in taste and consistency. For convenience, the very low-lipid sardine minced meats prepared through grinding or suspending processes were designated PMM-A and PMM-B, respectively, as an abbreviation for Purified Minced Meat (Nakayama et al., 1996). In the above-described study, the effects of added water on the flow property and the oil droplet size uniformity of fish meat emulsion were investigated for its quality improvement. In PMM-A emulsion with an oil ratio of 1.6 to sardine meat, ${ }^{1)}$ irreversible shear breakdown was observed at water ratio 0 , and shear-rate thixotropy was observed at a water ratio between 0.1 and 0.3 . All these samples were unstable and changed their flow property within 1-day storage. With an oil ratio of 1.1 , shear-rate thixotropy was exhibited at a water ratio between 0 and 0.3 . With no added water, the droplet size was nonuniform, and with some added water, it became uniform. However, the sample at water ratio 0.2 and 0.3 was not thick enough for a dressing-type food. Therefore in PMM-A emulsion, the sample with an oil ratio of 1.1 at water ratio 0.1 was superior with respect to flow property and droplet size uniformity. In PMM-B emulsion with an oil ratio of 1.1 or 1.6 , the irreversible shear breakdown and the droplet

\footnotetext{
1) In this report, the amount of emulsion constituents (e.g., egg-yolk, salad oil, vinegar, and added water) was described as a ratio to sardine meat. Hereinafter, only a ratio was described in order to avoid the repeated expression to sardine meat. In certain cases, the type difference of very low-lipid sardine minced meat was specified for the presentation of a ratio.
}

size nonuniformity were observed at water ratios 0 and 0.1 , and the thixotropy and the droplet size uniformity were observed at water ratios 0.2 and 0.3 . However, the sample at water ratio 0.3 was not thick enough as a dressing. Therefore, in the PMM-B emulsion, the sample with water ratio 0.2 at an oil ratio of 1.1 or 1.6 was superior with respect to flow property and droplet size uniformity.

Optical microscopy was useful in observing oil droplets larger than $5 \mu \mathrm{m}$ in diameter and taking a general wide-range view, but this technique was not suitable for determining the modal diameter of an oil droplet usually smaller than $5 \mu \mathrm{m}$ or observing the oil droplet surface and the myofibrillar matrix structure (Nakayama et al., 1996). In the present study, the oil droplet and the myofibrillar matrix were observed three-dimensionally at high magnification by scanning electron microscopy. The microstructures of the three superior samples, i.e., the PMM-A emulsion with an oil ratio of 1.1 at water ratio 0.1 and the PMM-B emulsions with an oil ratio of 1.1 or 1.6 at water ratio 0.2 , were compared.

Furthermore, the microstructures of the non-fish conventional emulsion and the fish meat emulsion were compared, and the different features of the fish meat emulsion were described regarding the role of the fish myofibrillar matrix.

\section{Materials and Methods}

Very low-lipid sardine minced meat Two types of very low-lipid sardine minced meat were prepared in the pilot plant of Taiyo Fishery Co., Ltd., in Nagasaki Prefecture, as previously reported (Nakayama et al., 1995, 1996).

Manufacturing of fish meat emulsion The fish meat emulsion was prepared from PMM-A or PMM-B as previously reported (Nakayama et al., 1992, 1995, 1996; Nakayama \& Tomita, 1996). Egg-yolk was used as an emulsifier. To adjust 


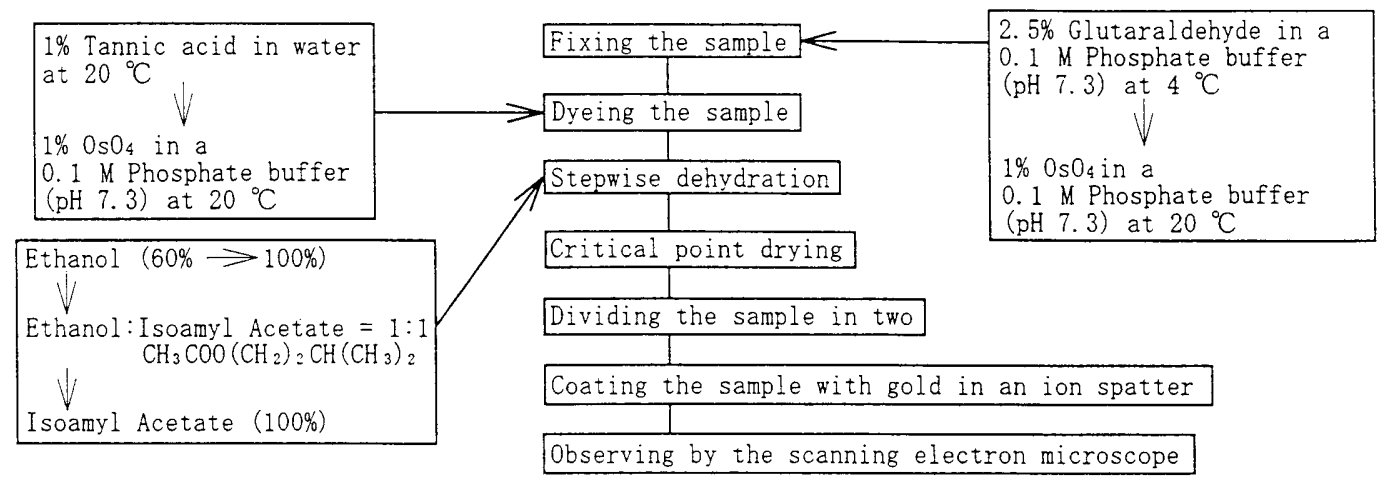

Fig. 1. Sample preparation for scanning electron microscopic observation.

the moisture content of the emulsion, the ratio of water added to PMM-A and PMM-B was set at 0.1 and 0.2 , respectively (Nakayama et al., 1994). With these water ratios, superior flow property was exhibited in the PMM-A and PMM-B emulsions (Nakayama et al., 1996). The constituent ratio of the fish meat emulsion was

\section{PMM-A or PMM-B : egg-yolk : salad oil : vinegar $=1: 0.7: 1.1$ or $1.6: 0.33$}

For an examination of the role of PMM, the non-fish conventional emulsion was prepared omitting PMM in this constituent ratio example. For an examination of the role of both PMM and salad oil, both PMM and salad oil were omitted.

Electron microscopy The sample preparation procedure for electron microscopic observation is shown in Fig. 1. From a 30-ml syringe with disposable pipette tip, several droplets of each emulsion, approximately $1.5 \mathrm{~mm}$ in diameter, were extruded onto the surface of a $0.1 \mathrm{M}$ phosphate buffer solution ( $\mathrm{pH} 7.3$ ) containing $2.5 \%$ glutaraldehyde at $4{ }^{\circ} \mathrm{C}$ and prefixed overnight. They were then postfixed with $1 \% \mathrm{OsO}_{4}$ in a $0.1 \mathrm{M}$ phosphate buffer $(\mathrm{pH} 7.3)$ at $20^{\circ} \mathrm{C}$. After dyeing by $1 \%$ tannic acid, the samples were dehydrated with ethanol and then with isoamyl acetate and dried by a critical point drier (HPC-2, Hitachi, Hitachinaka). Each sample globule was divided into two, and the divided surface was coated with gold in an ion spatter (JFC-1100, Jeol, Akishima). It was examined by a scanning electron microscope (JSM-T200, Jeol; S-4000, Hitachi; S-900, Hitachi).

Measurement of flow property The shear rate sweep measurement was carried out with a cone-and-plate rheometer (NRM-100, Nihon Rheology Kiki Co., Ltd., Funabashi) using a cone (top platen) having an angle of $3^{\circ}$ and a radius of $3.68 \mathrm{~cm}$. The plate (bottom platen) of the instrument was thermostated at $5 \pm 0.2^{\circ} \mathrm{C}$. The maximum shear rate was $200 \mathrm{~s}^{-1}$, and the sweep times taken for the increasing shear-rate curve and the decreasing one were both $100 \mathrm{~s}$. The power law equation was used to express the flow property during the shear-rate sweep measurement, as reported in the previous paper (Nakayama et al., 1996).

The flow property of a system composed of egg-yolk and vinegar belonged to a liquid-type, such as water. The coneand-plate rheometer was inapplicable to this system. Therefore, a coaxial cylinder-type viscometer (BL adapter, Tokyo
Keiki Co., Ltd., Tokyo) was used for the measurement of viscosity. The ratio of the inner cylinder's radius to that of the outer cylinder was 0.908 . The sample temperature was maintained at $5 \pm 0.2^{\circ} \mathrm{C}$ by a thermostated water bath surrounding the measuring head. The shear rates $2.28,4.56,11.4$, and $22.8 \mathrm{~s}^{-1}$ were applied to the sample for $20 \mathrm{~s}$ each.

\section{Results}

The arrangement of muscle fibers in fish is quite different from that in birds and mammals and is based on their necessity to flex their bodies for propulsion through water. The W-shaped segments standing on end are called myotomes, and they have one forward and two backward flexures. These myotomes are not perpendicular to the vertical midplane of the fish, but typically intercept this plane at sharp angles. Thus, if a cut is made at right angles to the central skeleton, multiple myotomes are exposed. The myotomes are one cell deep, and the muscle cells are roughly perpendicular to the surface of the myotome. The myotomes are connected one to another by thin layers of collagenous connective tissue called myosepta. Each myotome is composed of a large number of muscle fibers, generally shorter than those of mammalian muscles, that run obliquely to the main axis of the muscle system.

In the preparation of fish meat emulsion, PMM-A or PMM-B was homogenized and emulsified with the other ingredients by a Polytron homogenizer. In this process, myofibrils which constituted PMM-A or PMM-B were shattered into fragments. The fragments of myofibrils scattered among oil droplets were seen in the micrographs as shown in Fig. 2. The fragments having a diameter of $0.3-0.5$ $\mu \mathrm{m}$ and a length of $3.9-5.3 \mu \mathrm{m}$ were frequently seen in the PMM-B emulsion irrespective of oil ratio 1.1 or 1.6. Fragments of this size were not seen in the PMM-A emulsion, and the myofibrils were shattered to a much smaller size $0.1-0.4$ $\mu \mathrm{m}$ in diameter and $1.7-2.4 \mu \mathrm{m}$ in length. This difference in final fragment size was ascribed to the original fragment size in PMM-A and PMM-B, because the grinding process in the preparation of PMM-A produced the smaller size muscle fiber fragments, $2-20 \mu \mathrm{m}$ in diameter and $10^{-}-300 \mu \mathrm{m}$ in length, $60 \%$ of which was shorter than $125 \mu \mathrm{m}$, while the PMM-B contained fragments longer than $1000 \mu \mathrm{m}$ at $80 \%$.

In the PMM-A emulsion with water ratio 0.1 at oil ratio 1.1 , the modal diameter of the oil droplet was $0.55 \mu \mathrm{m}$, while 
Emulsifier : EGG-YOLK

Ingredient: $P M M-A$, salad oil, vinegar Ratio of salad oil to PMM-A : 1.1

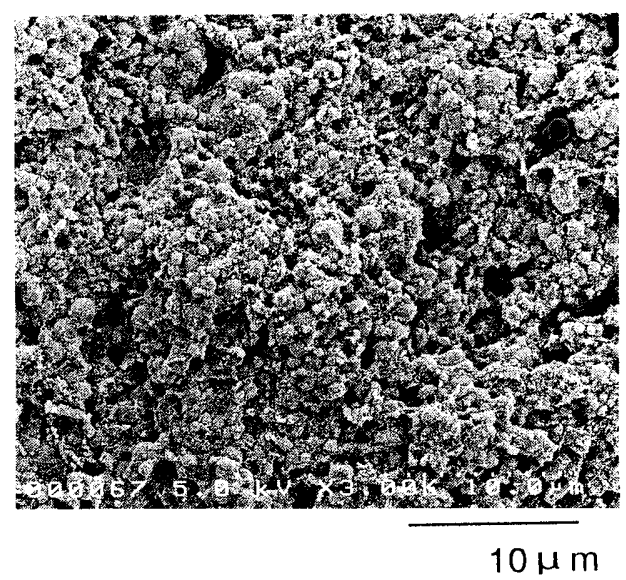

Emulsifier: EGG-YOLK

Ingredient: $P M M-B$, salad oil, vinegar Ratio of salad oil to PMM-B : 1.1

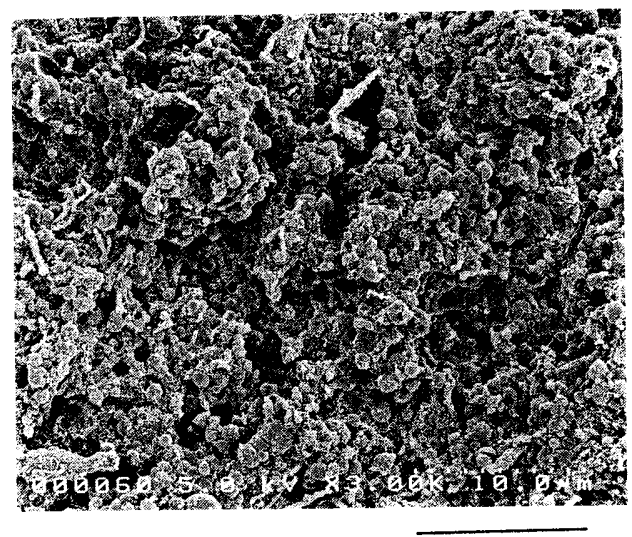

$10 \mu \mathrm{m}$

Emulsifier: EGG-YOLK

Ingredient: $P M M-B$, salad oil, vinegar Ratio of salad oil to PMM-B : 1.6

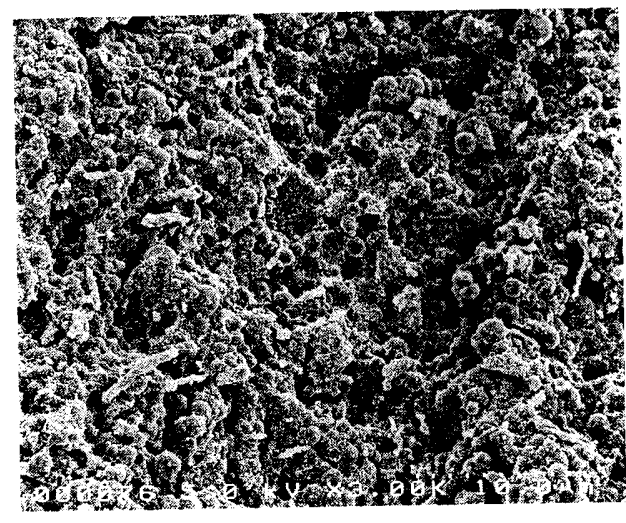

$10 \mu \mathrm{m}$

Fig. 2. Microscopic observation focused on fragments of myofibrils scattered among oil droplets. in the PMM-B emulsions with water ratio 0.2 at oil ratios 1.1 and 1.6 , it was 0.95 and $1.47 \mu \mathrm{m}$, respectively.

The oil droplets and matrix structure in fish meat emulsion and non-fish conventional emulsion are shown in Figs. 3 and 4. To detect the matrix structure difference between these emulsions and also to observe a possibly wider range, the microscopic observations were made at 7500 magnifications. The modal diameter of the oil droplet was $2.20 \mu \mathrm{m}$ in the non-fish emulsion, and larger than in the fish meat emulsions. A coarse honeycomb structure with densely packed thick walls was observed in the matrix of the non-fish emulsion. In this study, the optical microscopic modal diameters of fish meat emulsion previously reported (Nakayama et al, 1996) were reconfirmed at 7500 magnification, and the mesh size of the network structure made of fine filaments corresponded to the size of the oil droplets. The mesh size of the matrix network structure was the first-finest in the PMM-A emulsion with water ratio 0.1 at oil ratio 1.1 , and the second-finest in the PMM-B emulsion with water ratio 0.2 at oil ratio 1.1.

The oil droplets and myofibrillar matrix in the fish meat emulsion were observed at 20000 magnification to investigate the surface structure of the droplet and the dispersed state of the filaments, which are shown in Fig. 5. All the oil droplets were globular and possessed an almost-smooth surface to which the scattered yolk particles adhered. The modal diameters of the oil droplet in fish meat emulsions were reconfirmed also at 20000 magnification, and the mesh size of the network structure made of fine filaments corresponded to the size of the oil droplets. In the PMM-A and PMM-B emulsions at oil ratio 1.1, the continuous phase around the droplets was thick, while in the PMM-B emulsion at oil ratio 1.6, it was thin. This thin continuous phase between droplets in the latter emulsion coincided with the fact that the large oil droplets dispersed with high-oil content were surrounded by the same total amount of continuous phase.

A fragment of myofibril laid among oil droplets was observed at both 20000 and 30000 magnifications, as shown in Fig. 6. The myofibril generally $1 \mu \mathrm{m}$ in diameter became slender. A few occurrences of its bundle structure approximately $0.5 \mu \mathrm{m}$ in diameter at the thickest were detected in the left of Fig. 6, and such bundle structure was also seen in Fig. 2. Many usual occurrences of its filamentary component were observed in the right of Fig. 6. In the PMM-A emulsion at oil ratio 1.1, it was confirmed that the bundle structure was composed of filamentary components (left top of Fig. 6). The scattered yolk particles adhered to the surface of the bundle structure and also to each filamentary component which was spread apart from the bundle. The total amount of yolk particles which adhered to the surface of the filamentary component was smaller in the PMM-B emulsion at oil ratio 1.6 than in the PMM-A and PMM-B emulsions at oil ratio 1.1 , because the larger amount of yolk was expended on the surface of each large oil droplet with a high-oil content. The mesh size of the network structure made of filamentary components was finest in the PMM-A emulsion at oil ratio 1.1. In the PMM-B emulsion, it was finer at oil ratio 1.1 than at oil ratio 1.6. This mesh size of the network structure corresponded to the oil droplet size of each emulsion.

A bundle of filamentary components was observed at 
Oil droplets prefixed with $2.5 \%$ glutaraldehyde and postfixed with $1 \% \mathrm{OsO}_{4}$

\section{Emulsifier: EGG-YOLK}

Ingredient: $P M M-A$, salad oil, vinegar

Ratio of salad oil to PMM-A : 1.1

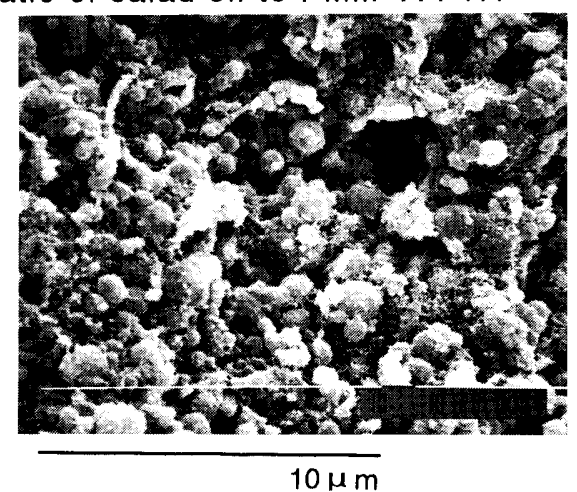

Emulsifier: EGG-YOLK

Ingredient: $P M M-B$, salad oil, vinegar

Ratio of salad oil to PMM-B : 1.1

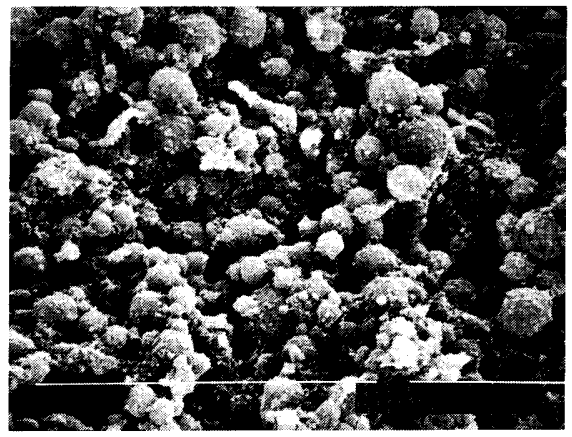

$10 \mu \mathrm{m}$

Emulsifier : EGG-YOLK

Ingredient: $P M M-B$, salad oil, vinegar

Ratio of salad oil to PMM-B : 1.6

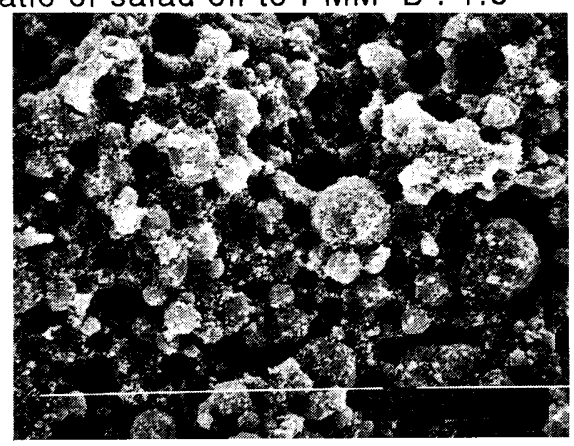

$10 \mu \mathrm{m}$
Myofibrillar matrix left after removal of oil droplets by ethanol treatment

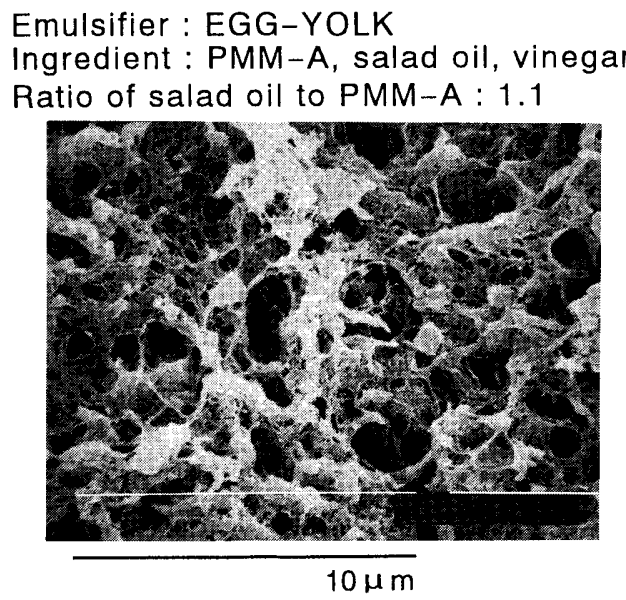

Emulsifier: $E G G-Y O L K$

Ingredient: $P M M-B$, salad oil, vinegar Ratio of salad oil to PMM-B: 1.1

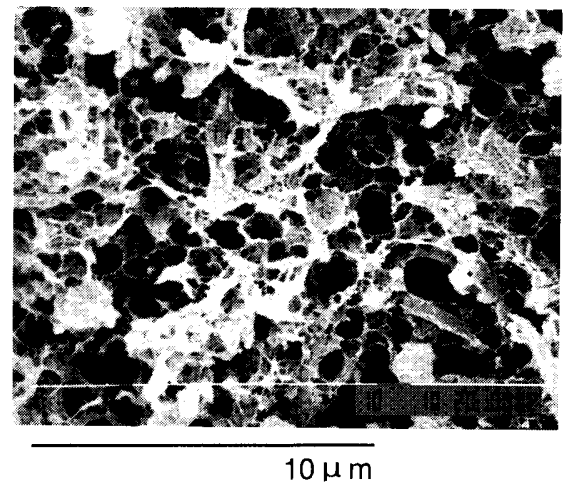

Emulsifier : EGG-YOLK

Ingredient: $P M M-B$, salad oil, vinegar Ratio of salad oil to PMM-B : 1.6

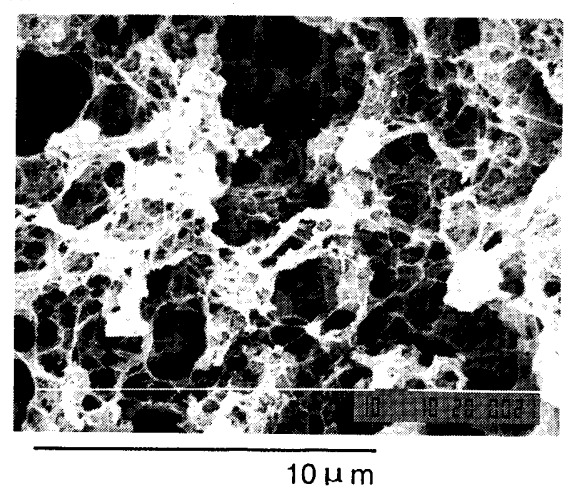

Fig. 3. Oil droplets and myofibrillar matrix in fish meat emulsion with addition of egg-yolk, observed at a low magnification.

60000 magnification to detect the detailed characteristic feature, as shown in Fig. 7. The diameters of a bundle and a filament were approximately $0.500 \mu \mathrm{m}$ (i.e., $500 \mathrm{~nm}$ ) and $0.035 \mu \mathrm{m}$ (i.e., $35 \mathrm{~nm}$ ), respectively (left of figure). A typical feature seen was that the filaments were spread apart from a bundle between oil droplets (middle of figure) and the network structure of filaments was formed (left of figure). From these spreading filaments and the network element, the diameter of a filament was also calculated as $0.035 \mu \mathrm{m}$. The diameters of thick and thin filaments in the muscle were 0.015 and $0.007 \mu \mathrm{m}$, respectively (Takahashi, 1983; Eskin, 1990a). Therefore it was concluded that the filament observed in this 


\section{Oil droplets prefixed with $2.5 \%$ glutaraldehyde and postfixed with $1 \% \mathrm{OsO}_{4}$}

\author{
Emulsifier: EGG-YOLK \\ Ingredient : salad oil, vinegar
}

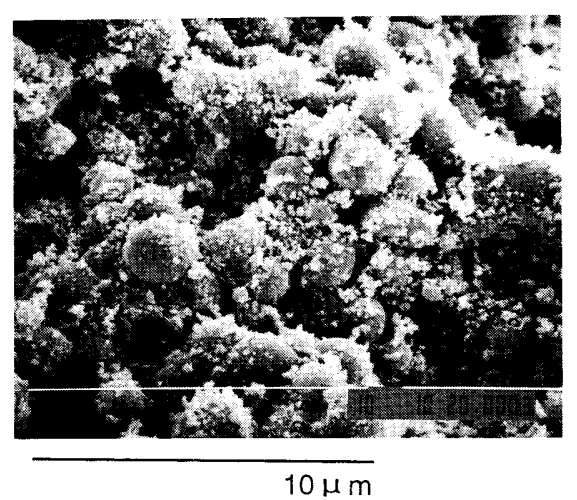

\section{Egg-yolk matrix left after removal \\ of oil droplets by ethanol treatment}

\author{
Emulsifier: EGG-YOLK \\ Ingredient: salad oil, vinegar
}

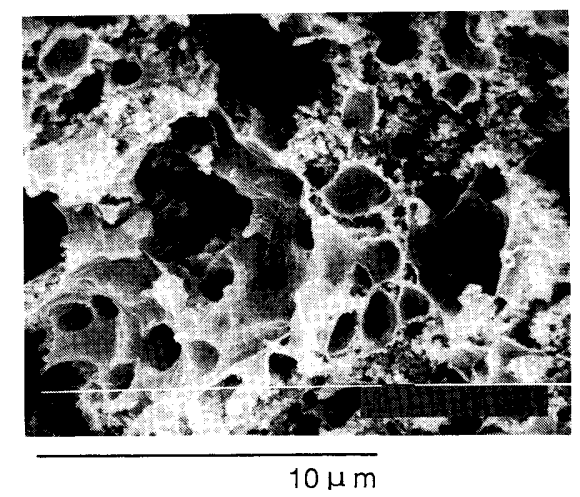

Fig. 4. Oil droplets and egg-yolk matrix in non-fish conventional emulsion with addition of egg-yolk, observed at a low magnification for comparison with fish meat emulsion.

study would be a thick filament surrounded by thin filaments.

The cross-bridge of filaments and the network structure were observed, but the filaments which adhered to the surface of the oil droplets were not detected. Therefore, some filaments existed in the vicinity of the oil droplets, and the other filaments surrounded them. However, there was not a strong binding between the filament and the oil droplet, and during shear application each of them could move independently.

At the high shear rate the oil droplets deformed and sometimes coalesced if the network structure could not hold them tightly by entanglement and the viscous environment. Because the egg-yolk was made of globular substances including emulsion stabilizers, such as low-density lipoprotein, myelin, and high-density lipoprotein (Powrie \& Nakai, 1985; Penfield \& Campbell, 1990), the non-fish conventional emulsion in which the yolk matrix structure (right of Fig. 4) was characterized by the coarse honeycomblike feature exhibited low viscosity as shown in Fig. 9. This emulsion with the yolk matrix exhibited a remarkable difference of shear stress between the increasing shear rate curve and the decreasing one over the shear rate range between 30 and $200 \mathrm{~s}^{-1}$; however, partial restoration (Nakagawa \& Kanbe, 1959) was observed in the decreasing shear rates below $20 \mathrm{~s}^{-1}$. Though the shear rate sweep measurement was repeated using the same sample after a rest period of $1 \mathrm{~h}$, a flow curve with more restoration was not obtained in the repetition of increasing shear rate. Therefore, an irreversible shear breakdown occurred.

In fish meat emulsion, the filamentary component of network structure was made of fibrous proteins, and the entanglement of fibrous protein polypeptide chains was included in the network structure. Therefore, the fish meat matrix was viscous and could hold the oil droplets tightly in it; the fish meat emulsion exhibited a weak trend of thix- otropy. When the shear rate sweep measurement was repeated using the same sample after a rest period of $1 \mathrm{~h}$, the flow curve with the perfect restoration, which was almost the same as the previous one, was obtained.

In the system composed of egg-yolk and vinegar (Fig. 8), the shear stresses at 2.28 and $22.8 \mathrm{~s}^{-1}$ were 0.159 and $1.436 \mathrm{~Pa}$ (i.e., viscosities 0.070 and $0.063 \mathrm{~Pa} \cdot \mathrm{s}$ ), respectively. A reference straight line of Newtonian flow was drawn from the origin of the coordinate to the shear stress plot $1.436 \mathrm{~Pa}$ at a shear rate of $22.8 \mathrm{~s}^{-1}$. All the shear stress plots existed approximately on this line in increasing and decreasing shear rates. The yield stress was not detected. Therefore, the flow property in the system composed of egg-yolk and vinegar was regarded in approximation as a Newtonian flow. In the non-fish conventional emulsion (left of Fig. 9), the participation of the dispersed oil in the above system brought about the pasty flow property of a mayonnaise type, and the shear stresses at 2.28 and $22.8 \mathrm{~s}^{-1}$ increased to 28.066 and $58.321 \mathrm{~Pa}$ (i.e., viscosities 12.310 and $2.557 \mathrm{~Pa} \cdot \mathrm{s}$ ), respectively. This viscosity increase was not sufficient to hold the oil droplets tightly during the shear application; as a result, an irreversible shear breakdown was induced by the occurrence of coalescence.

The non-oil PMM-A matrix (middle of Fig. 9) showed the shear stresses 142.272 and $173.402 \mathrm{~Pa}$ at 2.28 and $22.8 \mathrm{~s}^{-1}$, respectively, while the PMM-B matrix showed the shear stresses 125.670 and $157.175 \mathrm{~Pa}$ at these shear rates. These shear stress values were still higher than those of the non-fish conventional emulsion. The shear stress increased only gently with a further increase in shear rate and remained 245.414 and 241.013 Pa in the PMM-A and PMM-B matrixes, respectively, even at a maximum shear rate of $200 \mathrm{~s}^{-1}$.

In fish meat emulsion (right of Fig. 9), the shear stresses at 2.28 and $22.8 \mathrm{~s}^{-1}$ were 83.420 and $164.900 \mathrm{~Pa}$, respectively, in the PMM-A emulsion at oil ratio 1.1, while the values were 98.552 and 178.092 $\mathrm{Pa}$, respectively, in the PMM-B emulsion at oil ratio 1.1. The participation of oil droplets in the above 
Oil droplets prefixed with $2.5 \%$ glutaraldehyde and postfixed with $1 \% \mathrm{OsO}_{4}$

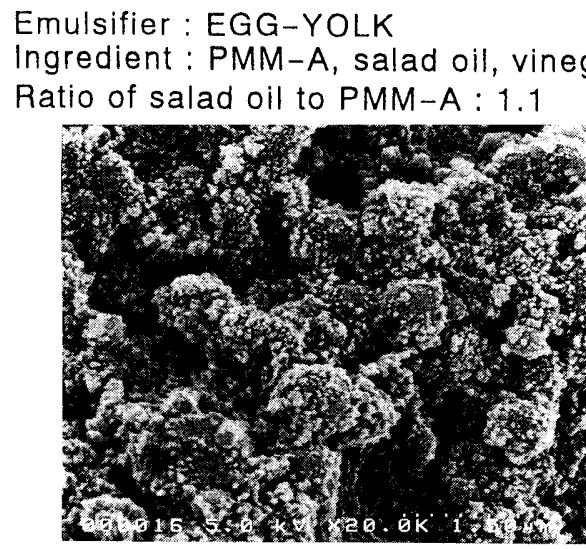

$1.5 \mu \mathrm{m}$

Emulsifier: EGG-YOLK

Ingredient: $P M M-B$, salad oil, vinegar Ratio of salad oil to $P M M-B: 1.1$

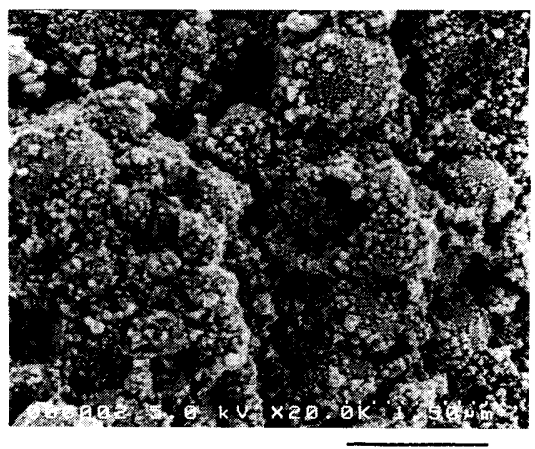

$1.5 \mu \mathrm{m}$

Emulsifier: EGG-YOLK

Ingredient: $P M M-B$, salad oil, vinegar

Ratio of salad oil to PMM-B : 1.6

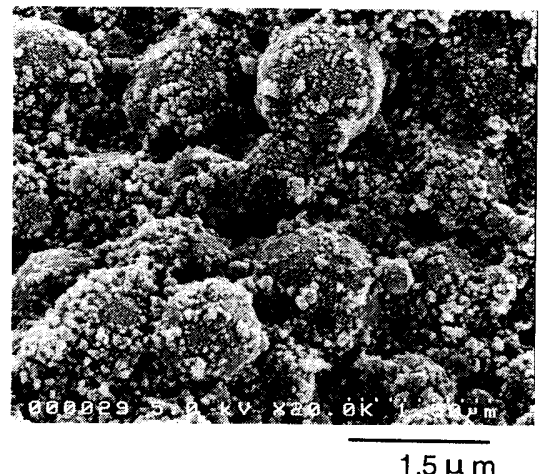

Myofibrillar matrix left after removal of oil droplets by ethanol treatment

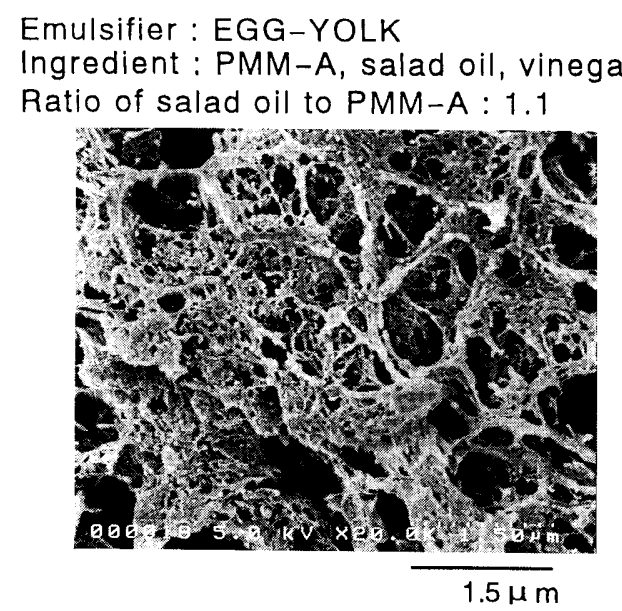

Emulsifier: EGG-YOLK

Ingredient: $P M M-B$, salad oil, vinegar

Ratio of salad oil to PMM-B : 1.1

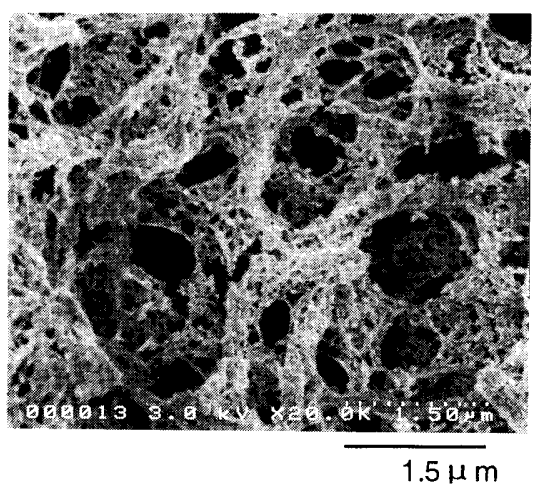

Emulsifier: EGG-YOLK

Ingredient: $P M M-B$, salad oil, vinegar

Ratio of salad oil to PMM-B : 1.6

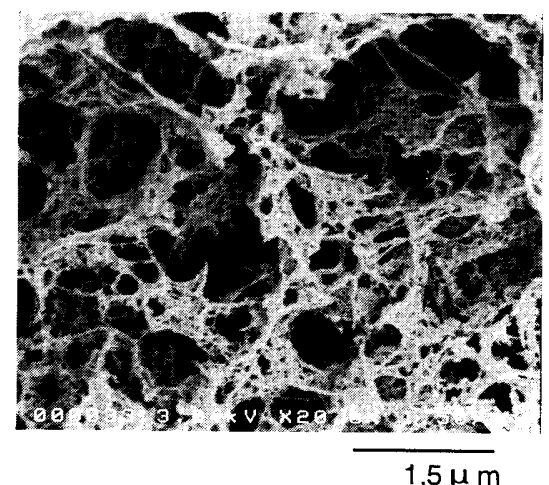

Fig. 5. Oil droplets and myofibrillar matrix in fish meat emulsion with addition of egg-yolk, observed at a high magnification.

PMM matrix decreased the shear stress at $2.28 \mathrm{~s}^{-1}$. However, the shear stress at $22.8 \mathrm{~s}^{-1}$ remained almost unchanged in the PMM-A emulsion and increased in the PMM-B emulsion. The shear stress at $200 \mathrm{~s}^{-1}$ remarkably increased to 320.100 and $318.160 \mathrm{~Pa}$ in these emulsions.

The addition of more oil (final oil ratio 1.6: right bottom of Fig. 9) did not bring about a further increase in shear stress; on the contrary, it decreased it. The shear stresses at 2.28, 22.8, and $200 \mathrm{~s}^{-1}$ were $77.600,155.200$, and $304.968 \mathrm{~Pa}$, respectively.

In fish meat emulsion, the network structure made of myofilaments (Figs. 3, 5, 6 and 7) showed a remarkable increase in viscosity (Fig. 9) and held the oil droplets tightly 


\section{Fragment of myofibril among oil droplets}

Emulsifier : EGG-YOLK

Ingredient: $P M M-A$, salad oil, vinegar

Ratio of salad oil to PMM-A : 1.1

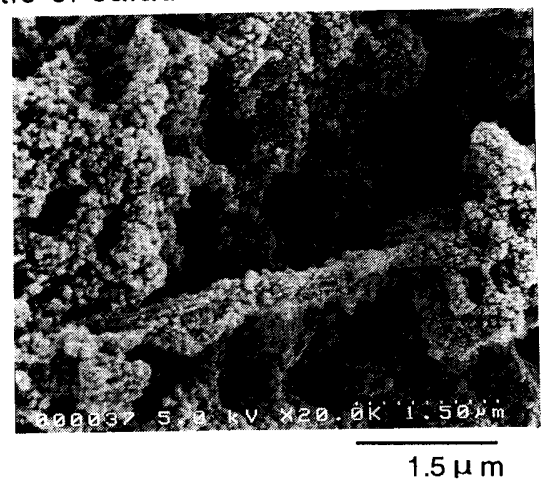

Emulsifier: EGG-YOLK

Ingredient: $P M M-B$, salad oil, vinegar Ratio of salad oil to PMM-B : 1.1

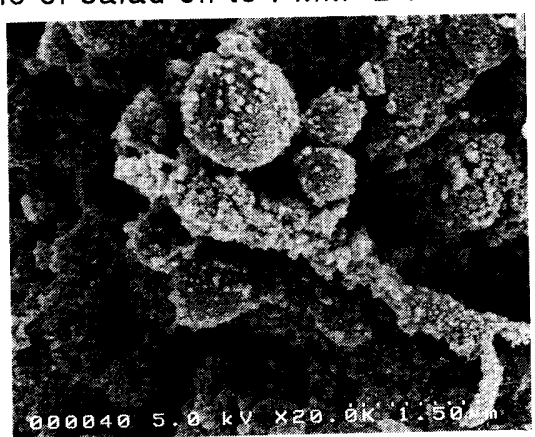

$1.5 \mu \mathrm{m}$

Emulsifier: $E G G-Y O L K$

Ingredient: $P M M-B$, salad oil, vinegar

Ratio of salad oil to PMM-B : 1.6

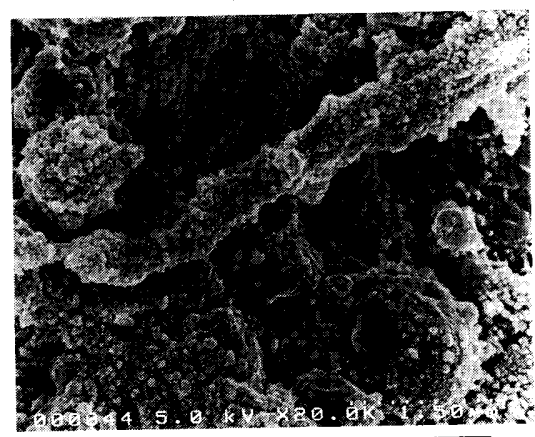

$1.5 \mu \mathrm{m}$

\section{Cross-bridge of filaments between oil droplets}

Emulsifier: $E G G-Y O L K$
Ingredient : PMM-A, salad oil, vinegar
Ratio of salad oil to PMM-A : 1.1

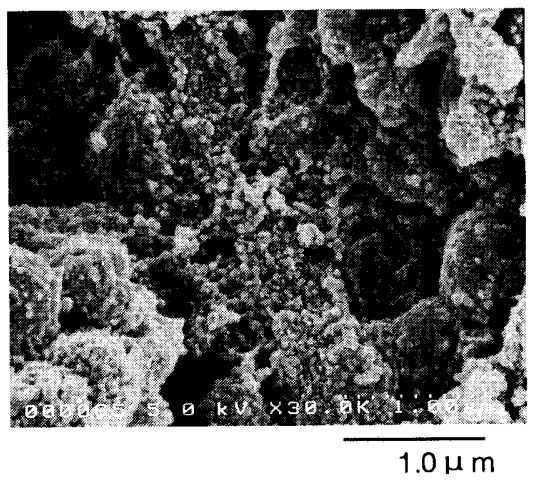

Emulsifier: EGG-YOLK

Ingredient: $P M M-B$, salad oil, vinegar

Ratio of salad oil to PMM-B : 1.1

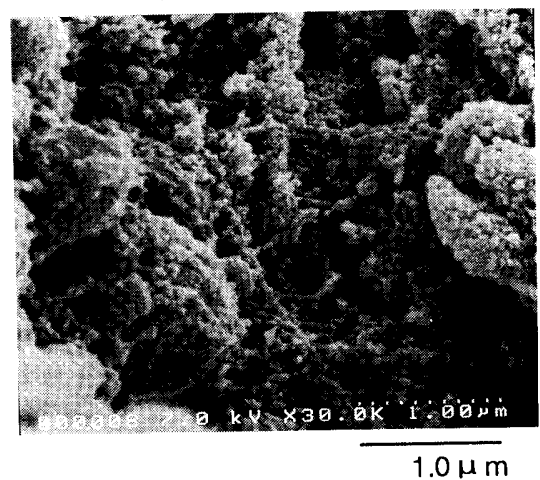

Emulsifier : EGG-YOLK

Ingredient: $P M M-B$, salad oil, vinegar

Ratio of salad oil to PMM-B : 1.6

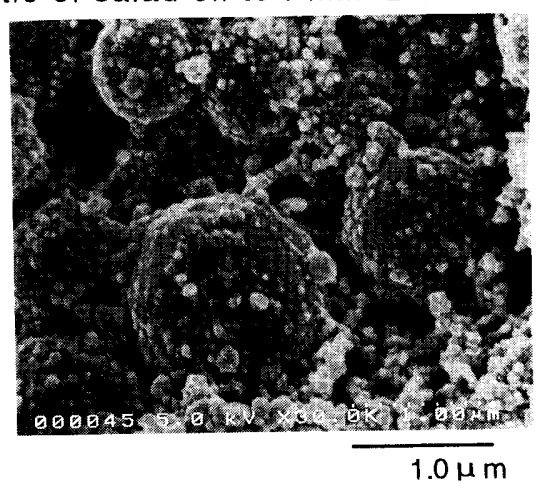

Fig. 6. Fragmented myofibril and its filamentary components among oil droplets observed at a high magnification.

during the application of high shear rates to prevent coalescence.

\section{Discussion}

Microstructure required for shearstability The lesser content of stroma protein (Hultin, 1985; Eskin, 1990b) and the shorter length of muscle fiber (Rodger \& Wilding, 1990) are necessary to fully demonstrate the movement and migration in sea water flexing the fish body. These characteristics in fish muscle were related to the ease in the deformation of the protein network in the fish meat emulsion. Such fish protein network (Fig. 7) easily accommodated its structure in 
Bundle of filaments

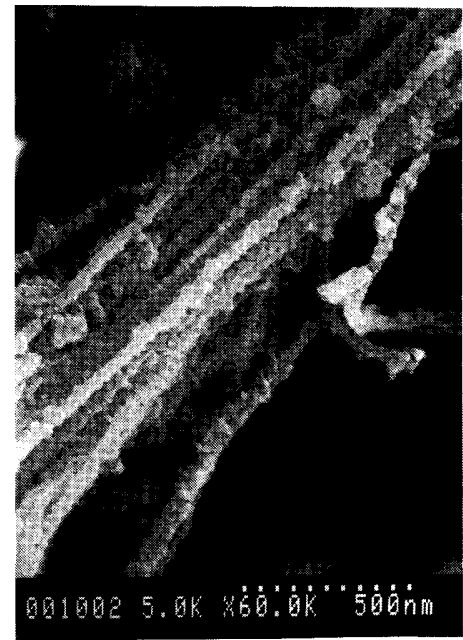

Filaments spread apart from bundle

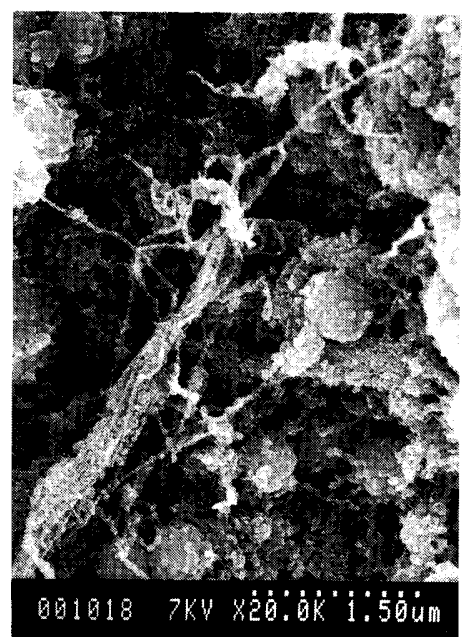

Network structure of filaments

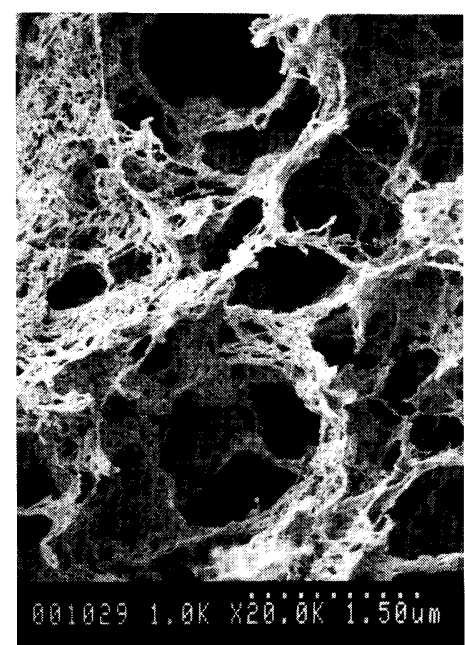

Fig. 7. Network formation process of filaments dispersed from fragmented myofibril.

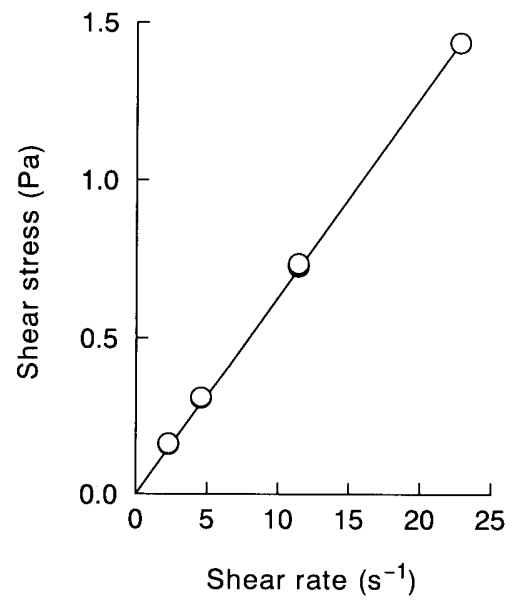

Fig. 8. Flow property of a system composed of egg-yolk and vinegar. The shear rates $2.28,4.56,11.4$, and $22.8 \mathrm{~s}^{-1}$ were applied to the same sample, and the increasing and decreasing shear rate curves were obtained. The front circles are plotted for the increasing shear rate curve, and the rear ones for the decreasing shear rate curve. A reference straight line of Newtonian flow is drawn from the origin of the coordinate to the shear stress plot $1.436 \mathrm{~Pa}$ at a shear rate of $22.8 \mathrm{~s}^{-1}$.

the increasing and decreasing shear rates, deforming and sliding the network components. When the flow ceased, the protein network easily recovered its original structure which existed before shear application. These properties were not demonstrated in the domestic animal meat emulsions, because the domestic animal meat contained more stroma proteins and was composed of much longer muscle fibers.

In PMM-A where the myofibrils were pulverized to a shorter size, the moisture content was $73.3 \%$, which was higher than the content in PMM-B. The optimum amount of water added to PMM-A was $10 \%$ for the emulsion preparation. In PMM-B where the myofibrils were coarsely pulverized and remained relatively long, the moisture content was $69.7 \%$. The $20 \%$ added water was required as an optimum for the emulsion preparation. The moisture contents in PMM-A and PMM-B became almost the same percentage of 75.7 and $74.8 \%$, respectively, by adding these amounts of water. Irrespective of fine or relatively coarse network structure formation in PMM-A and PMM-B based emulsions (Figs. 3 and 5), the flow property was almost the same (Fig. 9). Therefore it was concluded that the flow property was not affected by the mesh size of the network structure when the network formation was well made. The flow property was almost the same in the fine network of the PMM-A emulsion and the relatively coarse network of the PMM-B emulsion with the same amount of added oil (ratio 1.1); it was only a little affected by an increase in added oil (ratio 1.6). Namely, in the PMM-A emulsion with an oil ratio of 1.1 and the PMM-B emulsion with an oil ratio of 1.6 , the flow behavior index, $n$, of the up-curve was large, and the consistency index, $B$, was small (Table 1). This tendency was more distinct in the latter. These flow properties related to the result that the shear stress in the shear rate range between 20 and $125 \mathrm{~s}^{-1}$ increased more steeply than that of the PMM-B emulsion with an oil ratio of 1.1 (right of Fig. 9). This result was ascribed to the denser packing condition of oil droplets occasionally attained (Nakayama et al., 1996).

Efficacy and limitation in electron microscopic investigation The rheological parameters, which were obtained from the PMM-A emulsion with a water ratio between 0.1 and 0.3 at an oil ratio of 1.1 and the PMM-B emulsion with a water ratio 0.2 and 0.3 at an oil ratio of 1.1 or 1.6 , remained at almost the same values during 40 days of storage. The 


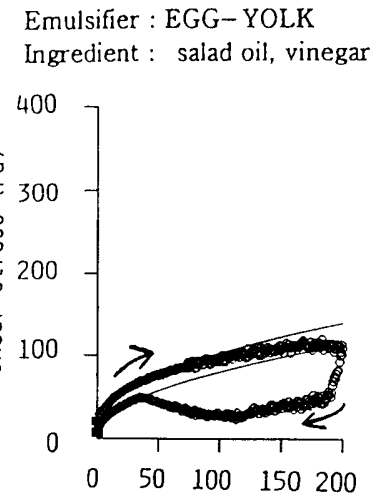

Shear rate $\left(s^{-1}\right)$
Emulsifier : EGG-YOLK

Ingredient : PMM-A, vinegar

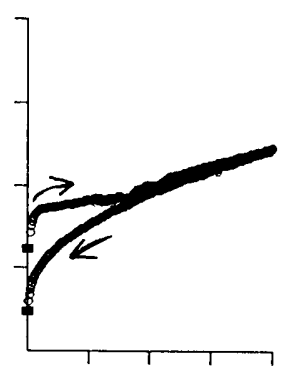

Emulsifier : EGG-YOLK

Ingredient : $\mathrm{PMM}-\mathrm{B}$, vinegar

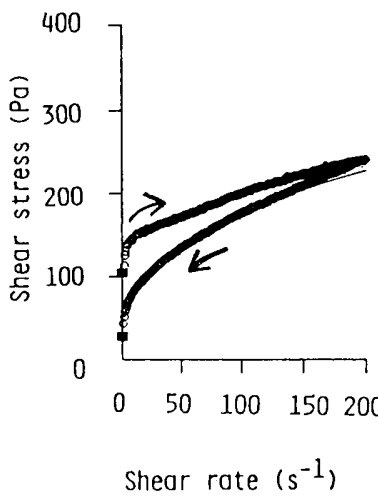

Emulsifier : EGG-YOLK

Ingredient : $\mathrm{PMM}-\mathrm{A}$, salad oil, vinegar

Ratio of salad oil to PMM-A : 1.1

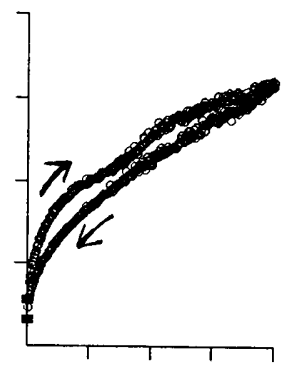

Emulsifier : EGG-YOLK

Ingredient : $\mathrm{PMM}-\mathrm{B}$, salad oil, vinegar

Ratio of salad oil to PMM-B : 1.1

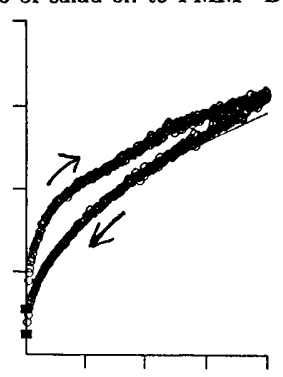

Emulsifier : EGG-YOLK

Ingredient : PMM- $B$, salad oil, vinegar

Ratio of salad oil to PMM-B : 1.6

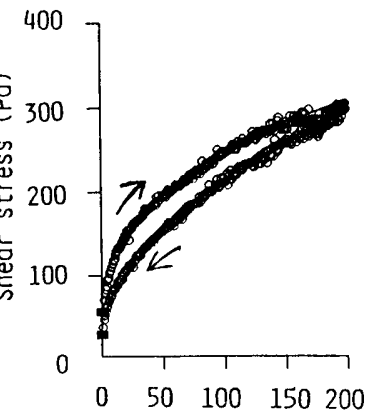

Shear rate $\left(s^{-1}\right)$

Fig. 9. Role of each ingredient in flow property of fish meat emulsion with addition of egg-yolk. For the rheological parameters, refer to Table 1.

parameter values, which were obtained from the emulsions with less water ratios, changed during the storage. These emulsions with less water ratios led to oil separation when extruded through the tip of a syringe for fixation in microscopy. In addition, these latter emulsions were so brittle after fixation that the smooth surfaces were not produced when dividing the sample in two for coating with gold. Therefore, they were not suitable for electron microscopy. In the former stable ones, the sample emulsions with water ratio 0.3 were too soft after being fixed for electron microscopy and broke down into pieces when dividing the sample in two for coating. They were not suitable for electron microscopy, either.

When our fish meat emulsions vacuum-packed in a plastic bag were kept in contact with ice, their cream color turned greenish yellow and some change in the flow property, detectable by our fingers, was induced. When they were frozen, the separation of oil phase was induced. Therefore, a cryo-method of scanning electron microscopy was not applicable to them.

A fixing and dewatering method of scanning electron microscopy could reconfirm the oil droplet size which was previously estimated in optical microscopy (Nakayama et al, 1996). The structure of the myofibrillar matrix surrounding the oil droplets might be slightly changed from the native state by a fixing and dewatering method. In our observation, however, the filaments in a myofibril, those filaments spread apart from their bundle, and the network structure of those filaments were precisely related to each other irrespective of the execution or non-execution of oil droplets extraction. Therefore, our findings were reliable and suggested that the general features of the matrix structure in fish meat emulsion 
Table 1. Rheological parameters obtained from each continuous flow curve of various dispersed systems.

\begin{tabular}{|c|c|c|c|c|c|c|c|}
\hline & \multicolumn{3}{|c|}{ Up-curve } & \multicolumn{3}{|c|}{ Down-curve } & \multirow{2}{*}{$\begin{array}{c}\text { Hysteresis loop } \\
\text { area surrounded } \\
\text { by increasing and } \\
\text { decreasing shear } \\
\text { rate curves } \\
\left(\mathrm{Pa} \cdot \mathrm{s}^{-1}\right)\end{array}$} \\
\hline & $\begin{array}{c}\text { Flow } \\
\text { behavior } \\
\text { index } \\
n\end{array}$ & $\begin{array}{c}\text { Yield } \\
\text { stress } \\
C \\
(\mathrm{~Pa})\end{array}$ & $\begin{array}{c}\text { Consistency } \\
\text { index } \\
B \\
\left(\mathrm{~Pa} \cdot \mathrm{s}^{n}\right)\end{array}$ & $\begin{array}{c}\text { Flow } \\
\text { behavior } \\
\text { index } \\
n\end{array}$ & $\begin{array}{l}\text { Yield } \\
\text { stress } \\
C \\
(\mathrm{~Pa})\end{array}$ & $\begin{array}{c}\text { Consistency } \\
\text { index } \\
B \\
\left(\mathrm{~Pa} \cdot \mathrm{s}^{n}\right)\end{array}$ & \\
\hline $\begin{array}{l}\text { Non-fish conventional } \\
\text { emulsion }\end{array}$ & 0.53 & 20.10 & 6.95 & 0.52 & 6.51 & 6.47 & 10200 \\
\hline $\begin{array}{l}\text { PMM-B matrix containing } \\
20 \% \text { added water }\end{array}$ & $\begin{array}{l}\text { non-applicable } \\
\text { to power law }\end{array}$ & 104.14 & non-computable & 0.44 & 27.54 & 19.08 & 5278 \\
\hline $\begin{array}{l}\text { PMM-A emulsion containing } \\
10 \% \text { added water } \\
\text { with an oil ratio of } 1.1\end{array}$ & 0.42 & 53.67 & 27.43 & 0.50 & 28.97 & 19.27 & 4984 \\
\hline $\begin{array}{l}\text { PMM-B emulsion containing } \\
20 \% \text { added water } \\
\text { with an oil ratio of } 1.1\end{array}$ & 0.35 & 58.47 & 37.19 & 0.47 & 22.88 & 21.12 & 6414 \\
\hline $\begin{array}{l}\text { PMM-B emulsion containing } \\
20 \% \text { added water } \\
\text { with an oil ratio of } 1.6\end{array}$ & 0.43 & 54.61 & 25.30 & 0.51 & 25.12 & 17.80 & 5699 \\
\hline $\begin{array}{l}\text { Q. P. HALF (semisolid-type } \\
\text { dressing) }\end{array}$ & 0.48 & 61.56 & 10.29 & 0.45 & 15.59 & 16.04 & 2505 \\
\hline
\end{tabular}

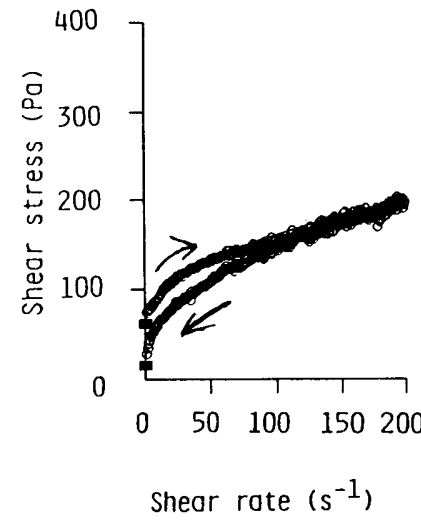

Fig. 10. Flow property of semisolid-type dressing "Q. P. HALF." For the rheological parameters, refer to Table 1.

were verified.

Pertinent flow property of our fish meat emulsion In French-type and American-type commercial mayonnaise (Nakayama et al, 1993), the shear rate sweep data up to $50 \mathrm{~s}^{-1}$ existed on the curve drawn by the power law equation. When the shear rate became larger than $50 \mathrm{~s}^{-1}$, the structure was broken down. The experimental points deviated from the power law curve, and large hysteresis loop areas, 12335 and $12843 \mathrm{~Pa} \cdot \mathrm{s}^{-1}$, were exhibited. Recently, a new mayonnaisetype product "Q. P. HALF" was developed as a healthy food with low oil content, half as much as the content in the French and American types. The structure instability during shear was improved in this product, and a small hysteresis loop area, $2505 \mathrm{~Pa} \cdot \mathrm{s}^{-1}$, was exhibited (Fig. 10 and Table 1). This improvement was attained by the use of a thickener (i.e., viscosity builder polysaccharide). Our fish meat emulsion also showed a small hysteresis loop area between 4984 and 6414 $\mathrm{Pa} \cdot \mathrm{s}^{-1}$ (Fig. 9 and Table 1). The addition of fish meat to the emulsion system improved the structure instability during the shear.

The system composed of egg-yolk and vinegar showed Newtonian viscosity (Fig. 8). The participation of the dispersed oil in this system brought about the pasty flow property of non-Newtonian viscosity. In this non-fish conventional emulsion, oil separation was induced after shear application up to $200 \mathrm{~s}^{-1}$. Its hysteresis loop area, $10200 \mathrm{~Pa}$. $\mathrm{s}^{-1}$, was large (Table 1). Three kinds of fish meat emulsion showed the very similar flow property of high viscosity in spite of different mesh size in their network structures, and they were stable without the oil separation in the shear and during storage. The new emulsified product "Q. P. HALF" contained almost the same amount of oil as our fish meat emulsions and prevented oil separation by the aid of a thickener. This "Q. P. HALF" and our fish meat emulsion exhibited a similar flow behavior index, $n$, and similar yield stress, $C$, in the increasing shear rates (Figs. 9 and 10; Table 1), and their hysteresis loop areas were both appropriately small. Therefore, our fish meat emulsion was recognized as a satisfactory emulsified food system.

Whether the network structure of the fish meat matrix in the fish meat emulsion was fine or relatively coarse, the emulsion was stable during the shear, if only the network structure was well formed holding the oil droplets tightly and avoiding their coalescence. Therefore, fish meat emulsion containing such fish meat network structure was more stable during the shear than the non-fish conventional emulsion containing the coarse honeycomb structure of the egg-yolk matrix with densely packed thick walls.

\section{References}

Eskin, N.A.M. (1990a). "Biochemistry of Foods," 2nd ed. Academic Press, San Diego, p. 7.

Eskin, N.A.M. (1990b). "Biochemistry of Foods," 2nd ed. Academic 
Press, San Diego, p. 10.

Hultin, H.O. (1985). Characteristics of muscle tissue. In "Food Chemistry," 2nd ed., ed. by O.R. Fennema. Marcel Dekker, New York, p. 730.

Nakagawa, T. and Kanbe, H. (1959). Shear stability, thixotropy, and irreversible shear breakdown. In "Rheology," 1st ed. Misuzu Shobou, Tokyo, pp. 456-462 (in Japanese).

Nakayama, T. and Tomita, H. (1996). Electron microscopic observation of fish meat emulsion with addition of egg-yolk. Presented at the 43rd Annual Conference of the Japanese Society for Food Science and Technology, Sendai, March 27-29, p. 132.

Nakayama, T., Oka, T., Ooi, A., Nonaka, M., Hirata, F. and Saeki, H. (1992). New emulsified product from sardine meat preparation with very low lipid content. J. Jpn. Soc. Food Sci. Technol., 39, 45I-456.

Nakayama, T., Tozaki, Y., Wakabayashi, A. and Ooi, A. (1993). Effect of yolk and white on shear-stable emulsion preparation from fish. J. Jpn. Soc. Food Sci. Technol., 40, 194-204.

Nakayama, T., Tomita, H., Murase, M. and Ooi., A. (1994). Effect of added water amount and yolk white ratio on flow property of fish emulsion. Presented at the 4lst Annual Conference of the Japanese Society for Food Science and Technology, Tokyo, March 28-30, p.
128.

Nakayama, T., Tomita, H. and Ooi, A. (1995). Relationship between change in flow property and coalescence of oil droplets in fish meat emulsion with addition of egg-white. Food Sci. Technol. Int., 1, 8288.

Nakayama, T., Murase, M., Tomita, H. and Ooi, A. (1996). Effect of added water on flow property and droplet size of fish meat emulsion with egg-yolk. Food Sci. Technol. Int., 2, 187-194.

Penfield, M.P. and Campbell, A.M. (1990). Eggs. In "Experimental Food Science," 3rd ed. Academic Press, San Diego, pp. 130-133.

Powrie, W.D. and Nakai, S. (1985). Characteristics of edible fluids of animal origin: eggs. In "Food Chemistry," 2nd ed., ed. by O.R. Fennema. Marcel Dekker, New York, pp. 837-855.

Rodger, G.W. and Wilding, P. (1990). Muscle proteins. In "Food Gels," 1st ed., ed. by P. Harris. Elsevier Science Publishers, London, pp. 364-365.

Takahashi, K. (1983). Property of meat proteins. In "Science of Food Proteins - Chemical Characteristics and Food Property -," 1st ed. ed. by F. Yamauchi. Shokuhin Shizai Kenkyukai, Tokyo, pp. 169180 (in Japanese) 